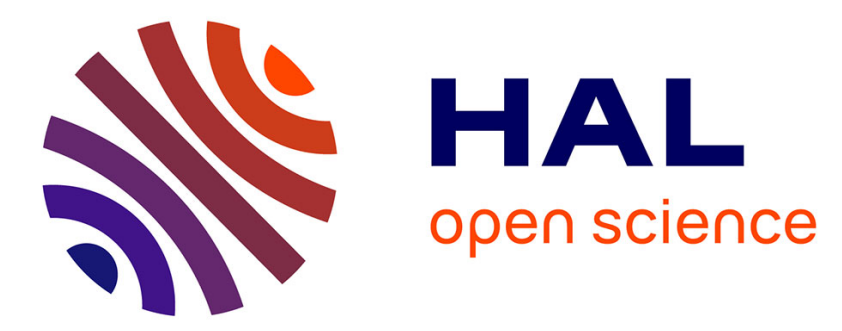

\title{
Robust sampled-data control: An input delay approach
} Emilia Fridman, Alexandre Seuret, Jean-Pierre Richard

\section{To cite this version:}

Emilia Fridman, Alexandre Seuret, Jean-Pierre Richard. Robust sampled-data control: An input delay approach. John Chiasson and Jean-Jacques Loiseau. Application of time delay systems, 352, Springer, pp.315-327, 2007, LNCIS, Lecture Notes in Control and Information Sciences, 978-3-54049555-0. inria-00179843v2

\section{HAL Id: inria-00179843 https://hal.inria.fr/inria-00179843v2}

Submitted on 27 Nov 2007

HAL is a multi-disciplinary open access archive for the deposit and dissemination of scientific research documents, whether they are published or not. The documents may come from teaching and research institutions in France or abroad, or from public or private research centers.
L'archive ouverte pluridisciplinaire HAL, est destinée au dépôt et à la diffusion de documents scientifiques de niveau recherche, publiés ou non, émanant des établissements d'enseignement et de recherche français ou étrangers, des laboratoires publics ou privés. 


\title{
Robust Sampled-Data Control: An Input Delay Approach
}

\author{
Emilia Fridman ${ }^{1}$, Alexandre Seuret ${ }^{2}$, and Jean-Pierre Richard ${ }^{2}$ \\ 1 Department of Electrical Engineering-Systems Tel-Aviv University, Tel-Aviv 69978, \\ Israel. \\ emilia@eng.tau.ac.il \\ 2 LAIL UMR 8021 Ecole Centrale de Lille, 59651 Villeneuve d'ascq cedex \\ seuret.Alexandre, jean-pierre.richard@ec-lille.fr
}

\section{Introduction}

Modelling of continuous-time systems with digital control in the form of continuous-time systems with delayed control input was introduced by Mikheev, Sobolev \& Fridman [19], Astrom \& Wittenmark [1] and further developed by Fridman (1992). The digital control law may be represented as delayed control as follows:

$$
u(t)=u_{d}\left(t_{k}\right)=u_{d}\left(t-\left(t-t_{k}\right)\right)=u_{d}(t-\tau(t)), \quad t_{k} \leq t<t_{k+1}, \tau(t)=t-t_{k},
$$

where $u_{d}$ is a discrete-time control signal and the time-varying delay $\tau(t)=t-t_{k}$ is piecewise-linear with derivative $\dot{\tau}(t)=1$ for $t \neq t_{k}$. Moreover, $\tau \leq t_{k+1}-t_{k}$. Based on such a model, for small enough sampling intervals $t_{k+1}-t_{k}$ asymptotic approximations of the trajectory [19] and of the optimal solution to the sampleddata LQ finite horizon problem [7] were constructed.

Since the middle of 90's years of the last century different LMI conditions for robust stability of linear systems with uncertain, but bounded constant delay with a given upper bound have been derived (see e.g. [18],[17] and [20]). For systems with time-varying delays such conditions were obtained via LyapunovKrasovskii functionals in the case where the derivative of the delay is less than one (see e.g. [15]). The stability issue in the cases of time-varying delay without any restrictions on the derivative of the delay has been treated mainly via Lyapunov-Razumikhin functions, which usually lead to conservative results (see e.g. [14],[16],[20] and [13]). Only recently for the first time this case was treated by Lyapunov-Krasovskii technique [11]. This became possible due to a new descriptor model representation of the delay system introduced by Fridman [8].

The main approach to the sampled-data robust stabilization problem (see e.g. [6], [21]) is based on the lifting technique ([2],[26]) in which the problem is transformed to equivalent finite-dimensional discrete problem. However, this approach does not work in the cases with uncertain sampling times or uncertain system matrices. In the present paper we suggest a new approach to the robust sampled-data stabilization. We find a solution by solving the problem for 
a continuous-time system with uncertain but bounded (by the maximum sampling interval) time-varying delay in the control input. We verify that the LMI sufficient conditions for stability of [11] are valid also in the case of piecewisecontinuous delay and derive LMIs for the feedback gain. The conditions which we obtain are robust with respect to different samplings with the only requirement that the maximum sampling interval is not greater than $h$. As a by-product we show that for $h \rightarrow 0$ the conditions coincide with the necessary and sufficient conditions for the continuous-time stabilization. Such convergence in $\mathrm{H}_{2}$ framework and related results were proved in [19], [4], [7], [22], [25] and [21].

For the first time the new approach allows to develop different robust control methods for the case of sampled-data control. The LMIs are affine in the system matrices and thus for the systems with polytopic type uncertainty the quadratic stabilization conditions, where the common Lyapunov functional for different vertices of the polytope is used, readily follow. We derive a parameter dependent solution, where different Lypunov functionals are used for different vertices by modifying results of (Fridman \& Shaked, 2003). We also consider the regional stabilization by sampled-data saturated state-feedback, where we give an estimate on the domain of attraction. For continuous-time stabilization of state-delayed systems by saturated-feedback see e.g. [5], [24], [3] and [10].

Notation: Throughout the paper the superscript ' $T$ ' stands for matrix transposition, $\mathcal{R}^{n}$ denotes the $n$ dimensional Euclidean space with vector norm $|\cdot|$, $\mathcal{R}^{n \times m}$ is the set of all $n \times m$ real matrices, and the notation $P>0$, for $P \in \mathcal{R}^{n \times n}$ means that $P$ is symmetric and positive definite. Given $\bar{u}=\left[\bar{u}_{1}, \ldots, \bar{u}_{m}\right]^{T}, 0<$ $\bar{u}_{i}, i=1, \ldots, m$, for any $u=\left[u_{1}, \ldots, u_{m}\right]^{T}$ we denote by $\operatorname{sat}(u, \bar{u})$ the vector with coordinates $\operatorname{sign}\left(u_{i}\right) \min \left(\left|u_{i}\right|, \bar{u}_{i}\right)$. By stability of the system we understand the asymptotic stability of it.

\section{Sampled-Data Stabilization of Systems with Polytopic Type Uncertainty}

\subsection{Problem Formulation}

Consider the system

$$
\dot{x}(t)=A x(t)+B u(t),
$$

where $x(t) \in \mathcal{R}^{n}$ is the state vector, $u(t) \in \mathcal{R}^{m}$ is the control input.

We are looking for a piecewise-constant control law of the form $u(t)=$ $u_{d}\left(t_{k}\right), t_{k} \leq t<t_{k+1}$, where $u_{d}$ is a discrete-time control signal and $0=t_{0}<t_{1}<$ $\cdots<t_{k}<\cdots$ are the sampling instants. Our objective is to find a state-feedback controller given by

$$
u(t)=K x\left(t_{k}\right), t_{k} \leq t<t_{k+1},
$$

which stabilizes the system.

We represent a piecewise-constant control law as a continuous-time control with a time-varying piecewise-continuous (continuous from the right) delay 
$\tau(t)=t-t_{k}$ as given in (1). We will thus look for a state-feedback controller of the form:

$$
u(t)=K x(t-\tau(t)) .
$$

Substituting (4) into (2), we obtain the following closed-loop system:

$$
\dot{x}(t)=A x(t)+B K x(t-\tau(t)), \quad \tau(t)=t-t_{k}, t_{k} \leq t<t_{k+1} .
$$

We assume that

A1 $t_{k+1}-t_{k} \leq h \forall k \geq 0$.

From A1 it follows that $\tau(t) \leq h$ since $\tau(t) \leq t_{k+1}-t_{k}$. We will further consider (5) as the system with uncertain and bounded delay.

\subsection{Stability of the Closed-Loop System}

Similarly to [11], where the continuous delay was considered, we obtain for the case of piecewise-continuous delay the following result:

Lemma 1. Given a gain matrix $K$, the system (5) is stable for all the samplings satisfying $A 1$, if there exist $n \times n$ matrices $0<P_{1}, P_{2}, P_{3}, Z_{1}, Z_{2}, Z_{3}$ and $R>0$ that satisfy the following LMIs:

$$
\Psi_{1}<0, \quad \text { and } \quad\left[\begin{array}{cc}
R[0 & \left.K^{T} B^{T}\right] P \\
* & Z
\end{array}\right] \geq 0
$$

where

$$
\begin{aligned}
& P=\left[\begin{array}{cc}
P_{1} & 0 \\
P_{2} & P_{3}
\end{array}\right], \quad Z=\left[\begin{array}{cc}
Z_{1} & Z_{2} \\
* & Z_{3}
\end{array}\right], \quad \Psi_{1}=\Psi_{0}+h Z+\left[\begin{array}{cc}
0 & 0 \\
0 & h R
\end{array}\right], \\
& \Psi_{0}=P^{T}\left[\begin{array}{cc}
0 & I \\
A+B K & -I
\end{array}\right]+\left[\begin{array}{cc}
0 & I \\
A+B K & -I
\end{array}\right]^{T} P .
\end{aligned}
$$

Proof is based on the following descriptor representation of (5) [8]:

$$
\dot{x}(t)=y(t), \quad 0=-y(t)+(A+B K) x(t)-B K \int_{t-\tau(t)}^{t} y(s) d s,
$$

which is valid in the case of piecewise-continuous delay $\tau(t)$ for $t \geq 0$. Given a matrix $K$ and initial condition $x(t)=\phi(t)(t \in[-h, 0])$, where $\phi$ is a piecewise continuous function, $x(t)$ satisfies $(5)$ for $t \geq 0$ iff it satisfies (7). Note that the descriptor system (7) has no impulsive solutions since in $(7) y(t)$ is multiplied by the nonsingular matrix I [9].

We apply the Lyapunov-Krasovskii functional of the form:

$$
V(t)=V_{1}+V_{2},
$$

where

$$
\bar{x}(t)=\operatorname{col}\{x(t), y(t)\}, \quad E=\left[\begin{array}{rr}
I_{n} & 0 \\
0 & 0
\end{array}\right], \quad P=\left[\begin{array}{rr}
P_{1} & 0 \\
P_{2} & P_{3}
\end{array}\right], \quad P_{1}=P_{1}^{T}>0,
$$


and

$$
V_{1}=\bar{x}^{T}(t) E P \bar{x}(t), \quad V_{2}=\int_{-h}^{0} \int_{t+\theta}^{t} y^{T}(s) R y(s) d s d \theta,
$$

which satisfies the following inequalities

$$
a|x(t)|^{2} \leq V(t) \leq b \sup _{s \in[-h, 0]}|\bar{x}(t+s)|^{2}, \quad a>0, b>0 .
$$

Differentiating $V(t)$ along the trajectories of (7) for $t \geq h$ we find (see [11]) that

$$
\dot{V}(t)<x \overline{(t)}^{T} \Psi_{1} x \overline{(t)}<-c|x(t)|^{2}, \quad c>0,
$$

Provided that $(6 \mathrm{a}, \mathrm{b})$ hold. Integrating $(11)$ we have

$$
V(t)-V(h) \leq-c \int_{-h}^{t}|x(s)| d s
$$

and, hence, (10) yields $|x(t)|^{2} \leq V(t) / a \leq V(h) / a<b / a \sup _{s \in[-h, 0]} \mid \bar{x}(h+$ $s)\left.\right|^{2}$. Since $\sup _{s \in[-h, 0]}|\bar{x}(h+s)| \leq c_{1} \sup _{s \in[-h, 0]}|\phi(s)|, c_{1}>0$ (cf. Hale \& Lunel, 1993, p168) and thus $\dot{x}$, defined by the right-hand side of (5), satisfy $\sup _{s \in[-h, 0]}|x(h+s)| \leq c_{2} \sup _{s \in[-h, 0]}|\phi(s)|, c_{2}>0$, we obtain that

$$
|x(t)|^{2} \leq c_{3} \sup _{s \in[-h, 0]}|\phi(s)|^{2}, c_{3}>0 .
$$

Hence (5) is stable (i.e. $x(t)$ is bounded and small for small $\phi$ ). To prove asymptotic stability we note that $x(t)$ is uniformly continuous on $[0, \infty)$ (since $\dot{x}(t)$ defined by the right-hand side of ( 5 is uniformly bounded). Moreover, (12) yields that $|x(t)|^{2}$ is integrable on $[0, \infty)$. Then, by Barbalat's lemma, $x(t) \rightarrow 0$ for $t \rightarrow \infty$

Consider now the continuous state-feedback

$$
u(t)=K x(t)
$$

and the closed-loop system (2), (14)

$$
\dot{x}(t)=(A+B K) x(t) .
$$

It is clear that the stability of the latter system is equivalent to the stability of its equivalent descriptor form

$$
\dot{x}(t)=y(t), \quad 0=-y(t)+(A+B K) x(t),
$$

which coincides with (7) for $h=0$. It is well-known ([23]) that the stability of the latter system is equivalent to the condition $\Psi_{0}<0$.

If there exists $P$ of the form $(9 \mathrm{c}, \mathrm{d})$ which satisfies $\Psi_{0}<0$, then for small enough $h>0$ LMIs of Lemma 2.1 are feasible (take e.g. $Z=I_{2 n}$ and $R=$ $\left.\left[0 K^{T} B^{T}\right] P^{T} P\left[0 K^{T} B^{T}\right]^{T}\right)$. We, therefore, obtain the following result: 
Corollary 1. If the continuous-time state-feedback (14) stabilizes the linear system (2), then the sampled-data state-feedback (3) with the same gain $K$ stabilizes (2) for all small enough $h$.

In the case where the matrices of the system are not exactly known, we denote $\Omega=[A B]$ and assume that $\Omega \in \mathcal{C} o\left\{\Omega_{j}, j=1, \ldots N\right\}$, namely,

$$
\Omega=\sum_{j=1}^{N} f_{j} \Omega_{j} \quad \text { for some } \quad 0 \leq f_{j} \leq 1, \sum_{j=1}^{N} f_{j}=1,
$$

where the $\mathrm{N}$ vertices of the polytope are described by

$$
\Omega_{j}=\left[A^{(j)} B^{(j)}\right] .
$$

In order to guarantee the stability of (2) over the entire polytope one can use the result of Lemma 2.1 by applying the same matrices $P_{2}$ and $P_{3}$ for all the points in the polytope and solving $(6 \mathrm{a}, \mathrm{b})$ for the $\mathrm{N}$ vertices only. A quadratic stability type criterion is then obtained:

Corollary 2. Given a gain matrix $K$, the system (5) is stable, over the entire polytope $\Omega$, if there exist $n \times n$ matrices $0<P_{1}^{(j)}, P_{2}, P_{3}, Z_{1}^{(j)}, Z_{2}^{(j)}, Z_{3}^{(j)}$ and $R^{(j)}>0$ that satisfy the following LMIs:

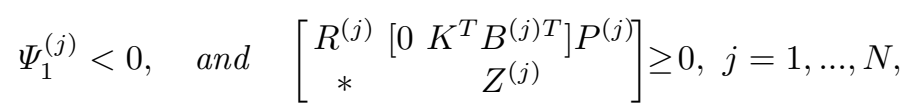

where

$$
\begin{aligned}
& P^{(j)}=\left[\begin{array}{rr}
P_{1}^{(j)} & 0 \\
P_{2} & P_{3}
\end{array}\right], \quad Z^{(j)}=\left[\begin{array}{cc}
Z_{1}^{(j)} & Z_{2}^{(j)} \\
* & Z_{3}^{(j)}
\end{array}\right], \quad \Psi_{1}^{(j)}=\Psi_{0}^{(j)}+h Z^{(j)}+\left[\begin{array}{cc}
0 & 0 \\
0 & h R^{(j)}
\end{array}\right], \\
& \Psi_{0}^{(j)}=P^{(j) T}\left[\begin{array}{cc}
0 & I \\
A^{(j)}+B^{(j)} K & -I
\end{array}\right]+\left[\begin{array}{cc}
0 & I \\
A^{(j)}+B^{(j)} K & -I
\end{array}\right]^{T} P^{(j)} .
\end{aligned}
$$

\subsection{Quadratic Stabilization}

LMIs of Lemma 2.1 are bilinear in $P$ and $K$. In order to obtain LMIs we use $P^{-1}$. It is obvious from the requirement of $0<P_{1}$, and the fact that in (6) $-\left(P_{3}+P_{3}^{T}\right)$ must be negative definite, that $P$ is nonsingular. Define

$$
P^{-1}=Q=\left[\begin{array}{cc}
Q_{1} & 0 \\
Q_{2} & Q_{3}
\end{array}\right] \text { and } \Delta=\operatorname{diag}\{Q, I\}
$$

Applying Schur formula to the term $h R$ in (6a), we multiply (6a,b) by $\Delta^{T}$ and $\Delta$, on the left and on the right, respectively. Denoting $\bar{R}=R^{-1}$ and $\bar{Z}=Q^{T} Z Q$ we obtain, similarly to [11], the following 
Theorem 1. The control law of (3) stabilizes (2) for all the samplings with the maximum sampling interval not greater than $h$ and for all the system parameters that reside in the uncertainty polytope $\Omega$, if there exist: $Q_{1}>0, Q_{2}^{(j)}, Q_{3}^{(j)}, \bar{R}$, $\bar{Z}_{1}^{(j)}, \bar{Z}_{2}^{(j)}, \bar{Z}_{3}^{(j)} \in \mathcal{R}^{n \times n}, \bar{Y} \in \mathcal{R}^{q \times n}$ that satisfy the following LMIs:

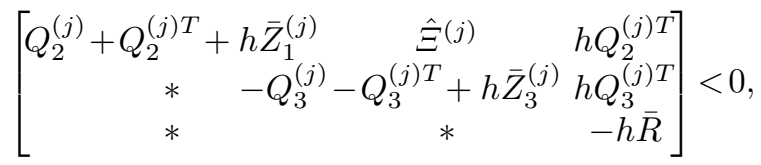

and nonlinear matrix inequalities

$$
\left[\begin{array}{ccc}
Q_{1} \bar{R}^{-1} Q_{1} & 0 & \bar{Y}^{T} B^{(j) T} \\
* & \bar{Z}_{1}^{(j)} & \bar{Z}_{2}^{(j)} \\
* & * & \bar{Z}_{3}^{(j)}
\end{array}\right] \geq 0
$$

where

$$
\hat{\Xi}^{(j)}=Q_{3}^{(j)}-Q_{2}^{(j) T}+Q_{1} A^{(j) T}+h \bar{Z}_{2}^{(j)}+\bar{Y}^{T} B^{(j) T}, \quad j=1,2, \ldots, N .
$$

The state-feedback gain is then given by

$$
K=\bar{Y} Q_{1}^{-1} .
$$

For solving (20) there exist two methods. The first uses the assumption

$$
\bar{R}=\epsilon Q_{1}, \quad \epsilon>0,
$$

and thus leads to $2 N$ LMIs with tuning parameter $\epsilon$ :

$$
\begin{gathered}
{\left[\begin{array}{cccc}
Q_{2}^{(j)}+Q_{2}^{(j) T}+h \bar{Z}_{1}^{(j)} & \hat{\Xi}^{(j)} & h Q_{2}^{(j) T} \\
* & -Q_{3}^{(j)}-Q_{3}^{(j) T}+h \bar{Z}_{3}^{(j)} & h Q_{3}^{(j) T} \\
* & & * & -\epsilon h Q_{1}
\end{array}\right]<0,} \\
\\
{\left[\begin{array}{ccc}
\epsilon Q_{1} & 0 & \epsilon \bar{Y}^{T} B^{(j) T} \\
* & \bar{Z}_{1}^{(j)} & \bar{Z}_{2}^{(j)} \\
* & * & \bar{Z}_{3}^{(j)}
\end{array}\right] \geq 0,}
\end{gathered}
$$

where $\hat{\Xi}^{(j)}$ and $j$ are given by $(21)$.

Similarly to Corollary 2.2 we can show that if the system (2) is quadratically stabilizable by a continuous-time state-feedback (14), then for all small enough $h$ the latter LMIs are feasible and the sampled-data state-feedback with the same gain stabilizes the system.

The second method for solving the matrix inequalities of Lemma 2.4 is based on the iterative algorithm developed recently by Gao and Wang (2003). This method is preferable in the cases of comparatively large $h$, since it leads to less conservative results. However it may take more computer time due to iterative 
process. In the sequel we shall adopt the first method for solving the matrix inequalities of Lemma 2.4 .

Example 1. We consider (2) with the following matrices:

$$
A=\left[\begin{array}{ll}
1 & 0.5 \\
0 & -1
\end{array}\right], \quad B=\left[\begin{array}{c}
1 \\
-1
\end{array}\right] .
$$

It is verified by using Theorem 2.4 that the system is stabilizable by a sampleddata state-feedback with the maximum sampling interval $h \leq 0.69$. Thus, for $h=0.69$ the resulting $K=\left[\begin{array}{ll}-1.048 & 0.2511\end{array}\right]$ (with $\epsilon=0.34$ ). Simulation results (for uniform samplings with the sampling interval less than 0.7 ) show that the closed-loop system is stable.

\subsection{Parameter Dependent Stabilization}

The requirement for the quadratic stabilization imposes a serious constraint on the solution, where the same matrices $Q_{1}$ should satisfy the matrix inequalities in all the vertices of the polytope. To alleviate this difficulty a parameter dependent solution with different matrices $Q_{1}^{(j)}$ was derived in [12] for the case of state delay. We modify the results of Corollary 4 of [12] for the case of input delay by assuming that $\bar{R}=\epsilon G_{1}$ and obtain

Theorem 2. Consider the system (2) and assume that its parameters lie in the polytope $\bar{\Omega}$. The system is stabilized, over the entire polytope $\Omega$, by the controller of (3), for all the samplings with the maximum sampling interval not greater than $h$, if for some tuning positive scalar parameters $\epsilon$ and $\alpha$ there exist $2 n \times 2 n$ matrices: $Q_{j}, G_{j}$ and $H_{j}$, of the form

$$
Q_{j}=\left[\begin{array}{cc}
Q_{1}^{(j)} & 0 \\
Q_{2}^{(j)} & Q_{3}^{(j)}
\end{array}\right], G_{j}=\left[\begin{array}{cc}
G_{1} & 0 \\
G_{2}^{(j)} & G_{3}^{(j)}
\end{array}\right], H_{j}=\left[\begin{array}{cc}
\alpha G_{1} & 0 \\
H_{2}^{(j)} & H_{3}^{(j)}
\end{array}\right] .
$$

and $\bar{Z}_{j}, j=1, \ldots, \bar{N}$, a $m \times n$ matrix $\bar{Y}$ and $n \times n$ matrix $\bar{R}$ that satisfy the following LMIs.

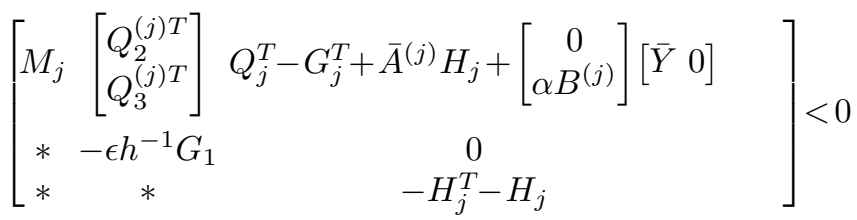

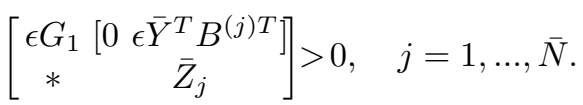

where

$$
M_{j}=G_{j}^{T} \bar{A}^{(j) T}+\bar{A}^{(j)} G_{j}+\left[\begin{array}{c}
\bar{Y}^{T} \\
0
\end{array}\right]\left[0 B^{(j) T}\right]+\left[\begin{array}{c}
0 \\
B^{(j)}
\end{array}\right]\left[\begin{array}{ll}
\bar{Y} & 0
\end{array}\right]+h \bar{Z}_{j}, \bar{A}^{(j)}=\left[\begin{array}{cc}
0 & I \\
A^{(j)} & -I
\end{array}\right] .
$$

The state-feedback gain that stabilizes the system over $\Omega$ is then given by $K=$ $\bar{Y} G_{1}^{-1}$. 
Example 2. We consider (2) with the following matrices taken from [10], where $h=0$ :

$$
A=\left[\begin{array}{ll}
0 & 1 \\
-1+g_{1}-.5
\end{array}\right], B=\left[\begin{array}{c}
-1+g_{2} \\
1
\end{array}\right]
$$

and where $\left|g_{1}\right| \leq 0.53$ and $\left|g_{2}\right| \leq 1$.7. It was shown in [12] that the system is not quadratically stabilizable by continuous state-feedback (i.e. for $h=0$ ). It is verified by using Theorem 2.5 that the system is stabilizable by a sampleddata state-feedback with the maximum sampling interval $h \leq 0.299$. Thus, for $h=0.299$ the resulting $K=[0.0821-0.1487]$ (with $\epsilon=3.56$ and $\alpha=1.1$ ). Simulation results (see e.g. Fig.1 for the case of $g_{1}=g_{1}(t)=0.53 \sin t$, $g_{2}=g_{2}(t)=1.7 \cos t, t_{k+1}-t_{k}=0.299 \forall k \geq 0$ and the initial condition $x(0)=\left[\begin{array}{ll}5 & -5\end{array}\right]^{T}$ ) show that the resulting closed-loop solutions converge to origin.

\section{Regional Stabilization by Sampled-Data Controller with Saturation}

\subsection{Problem Formulation}

Consider the system (2) with the sampled-data control law (3) which is subject to the following amplitude constraints

$$
\left|u_{i}(t)\right| \leq \bar{u}_{i}, \quad 0<\bar{u}_{i}, i=1, \ldots, m
$$

Denote by $x(t, x(0))$ the state trajectory of (2) with the initial condition $x(0) \in$ $R^{n}$. Then the domain of attraction of the origin of the closed-loop system (2), (3) is the set

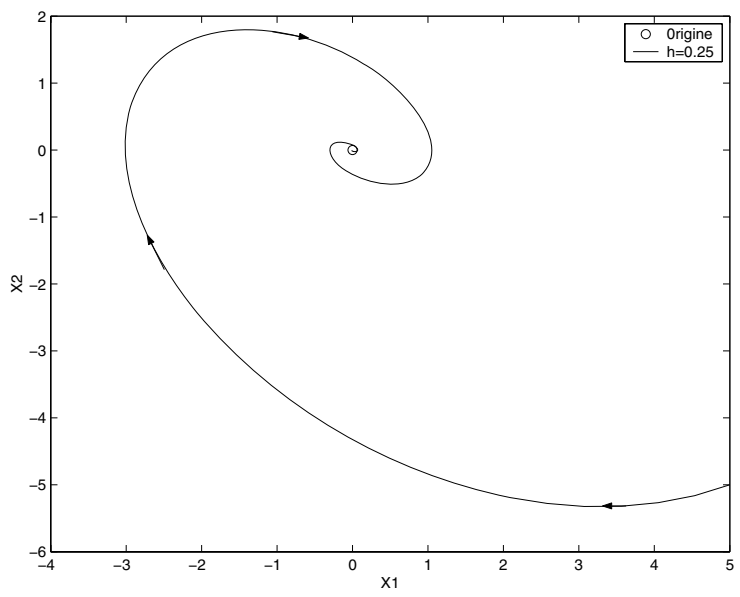

Fig. 1. State trajectories of the closed-loop system for $h=0.299, g_{1}=0.53 \sin t$, $g_{2}=1.7 \cos t$ and $x(0)=\left[\begin{array}{ll}5 & -5\end{array}\right]^{T}$ 


$$
\mathcal{A}=\left\{x(0) \in R^{n}: \lim _{t \rightarrow \infty} x(t, x(0))=0\right\} .
$$

We seek conditions for the existence of a gain matrix $K$ which leads to a stable closed-loop. Having met these conditions, a simple procedure for finding the gain $K$ should be presented. Moreover, we obtain an estimate $\mathcal{X}_{\beta} \subset \mathcal{A}$ on the domain of attraction, where

$$
\mathcal{X}_{\beta}=\left\{x(0) \in R^{n}: x^{T}(0) P_{1} x(0) \leq \beta^{-1}\right\},
$$

and where $\beta>0$ is a scalar and $P_{1}>0$ is an $n \times n$ matrix.

We represent the state-feedback in the delayed form

$$
u(t)=\operatorname{sat}(K x(t-\tau(t)), \bar{u}) .
$$

Reducing the original problem to the problem with input delay, we solve it by modifying derivations of [10], where the case of state delay was considered.

\subsection{A Linear System Representation with Polytopic Type Uncertainty}

Applying the control law of (29) the closed-loop system obtained is

$$
\dot{x}(t)=A x(t)+B \operatorname{sat}(K x(t-\tau(t)), \bar{u}), \tau(t)=t-t_{k}, t_{k} \leq t<t_{k+1} .
$$

Though the closed-loop system has a delay, we keep in mind that in the case of sampled-data control the initial condition is defined in the point $t=0$ and not on the segment $[-h, 0]$. That is why for the estimation of the domain of attraction we can restrict ourself to the following initial functions $\phi(s), s \in[-h, 0]$ :

$$
\phi(0)=x(0), \phi(s)=0, s \in[-h, 0) .
$$

Denoting the i-th row by $k_{i}$, we define the polyhedron

$$
\mathcal{L}(K, \bar{u})=\left\{x \in \mathcal{R}^{n}:\left|k_{i} x\right| \leq \bar{u}_{i}, i=1, \ldots, m\right\} .
$$

If the control and the disturbance are such that $x \in \mathcal{L}(K, \bar{u})$ then the system (30) admits the linear representation. Following [3], we denote the set of all diagonal matrices in $\mathcal{R}^{m \times m}$ with diagonal elements that are either 1 or 0 by $\Upsilon$, then there are $2^{m}$ elements $D_{i}$ in $\Upsilon$, and for every $i=1, \ldots, 2^{m} D_{i}^{-} \triangleq I_{m}-D_{i}$ is also an element in $\Upsilon$.

Lemma 2. [3] Given $K$ and $H$ in $\mathcal{R}^{m \times n}$. Then

$$
\operatorname{sat}(K x(t), \bar{u}) \in \mathcal{C} o\left\{D_{i} K x+D_{i}^{-} H x, i=1, \ldots, 2^{m}\right\}
$$

for all $x \in \mathcal{R}^{n}$ that satisfy $\left|h_{i} x\right| \leq \bar{u}_{i}, i=1, \ldots, 2^{m}$.

The following is obtained from Lemma 3.1. 
Lemma 3. Given $\beta>0$, assume that there exists $H$ in $\mathcal{R}^{m \times n}$ such that $\left|h_{i} x\right| \leq$ $\bar{u}_{i}$ for all $x(t) \in \mathcal{X}_{\beta}$. Then for $x(t) \in \mathcal{X}_{\beta}$ the system (30) admits the following representation.

$$
\dot{x}(t)=A x(t)+\sum_{j=1}^{2^{m}} \lambda_{j}(t) A_{j} x(t-\tau(t))
$$

where

$$
A_{j}=B\left(D_{j} K+D_{j}^{-} H\right) \quad j=1, \ldots, 2^{m}, \sum_{j=1}^{2^{m}} \lambda_{j}(t)=1,0 \leq \lambda_{j}(t), \forall 0<t,
$$

We denote

$$
\Omega_{\alpha}=\sum_{j=1}^{2^{m}} \lambda_{j} \Omega_{j} \quad \text { for all } \quad 0 \leq \lambda_{j} \leq 1, \sum_{j=1}^{2^{m}} \lambda_{j}=1
$$

where the vertices of the polytope are described by $\Omega_{j}=\left[A_{j}\right], \quad j=1, \ldots, 2^{m}$. The problem becomes one of finding $\mathcal{X}_{\beta}$ and a corresponding $H$ such that $\left|h_{i} x\right| \leq$ $\bar{u}_{i}, i=1, \ldots 2^{m}$ for all $x \in \mathcal{X}_{\beta}$ and that the state of the system

$$
\dot{x}(t)=A x(t)+A_{j} x(t-\tau(t)), \tau(t)=t-t_{k}, t_{k} \leq t<t_{k+1},
$$

remains in $\mathcal{X}_{\beta}$.

\subsection{Regional Stabilization}

Applying the descriptor model transformation and the Lyapunov-Krasovskii functional of (8) by using the first method for solving the stabilization matrix inequalities (with tuning parameter $\epsilon$ ), we obtain the following result:

Theorem 3. Consider the system (2) with the sampled-data control law (3) which is subject to the constraints (27). The system is stable with $\mathcal{X}_{\beta}$ inside the domain of attraction for all the samplings with the maximum sampling interval not greater than $h$, if there exist $0<Q_{1}, Q_{2}^{(j)}, Q_{3}^{(j)}, Z_{1}^{(j)}, Z_{2}^{(j)}, Z_{3}^{(j)} \in \mathcal{R}^{n \times n}$, $Y, G \in \mathcal{R}^{m \times n}$ and $\beta>0$ that satisfy the following set of inequalities:

$$
\begin{aligned}
& {\left[\begin{array}{ccc}
Q_{2}^{(j)}+Q_{2}^{T(j)}+h Z_{1}^{(j)} & \Sigma_{j} & h Q_{2}^{(j)} \\
* & -Q_{3}^{(j)}-Q_{3}^{T(j)}+h Z_{3}^{(j)} & h Q_{3}^{(j)} \\
* & * & -\epsilon h Q_{1}
\end{array}\right]<0, j=1, \ldots, 2^{m}} \\
& {\left[\begin{array}{ccc}
\epsilon Q_{1} & 0 & \epsilon\left(Y^{T} D_{j}+G^{T} D_{j}^{-}\right) B^{T} \\
* & Z_{1}^{(j)} & Z_{2}^{(j)} \\
* & * & Z_{3}^{(j)}
\end{array}\right] \geq 0} \\
& {\left[\begin{array}{cc}
\beta & g_{i} \\
* & \bar{u}_{i}^{2} Q_{1}
\end{array}\right] \geq 0, i=1, \ldots, m,}
\end{aligned}
$$

where

$$
\Sigma_{j}=Q_{3}^{(j)}-Q_{2}^{T(j)}+Q_{1} A^{T}+\left(Y^{T} D_{j}+G^{T} D_{j}^{-}\right) B^{T}+h Z_{2}^{(j)} .
$$

The feedback gain matrix which stabilizes the system is given by $K=Y Q_{1}^{-1}$. 
Proof: For $V$ given by (8) conditions are sought to ensure that $\dot{V}<0$ for any $x(t) \in \mathcal{X}_{\beta}$. As in [10], the inequalities (37) guarantee that $\left|h_{i} x\right| \leq \bar{u}_{i}, \forall x \in$ $\mathcal{X}_{\beta}, i=1, \ldots, m$, where $g_{i} \triangleq h_{i} Q_{1}, i=1, \ldots, m$ and $Q_{1} \triangleq P_{1}^{-1}$, and the polytopic system representation of (35) is thus valid. Moreover, $(36 \mathrm{a}, \mathrm{b})$ guarantee that $\dot{V}<0$.

From $\dot{V}<0$ it follows that $V(t)<V(0)$ and therefore for the initial conditions of the form (31)

$$
x^{T}(t) P_{1} x(t) \leq V(t)<V(0)=x^{T}(0) P_{1} x(0) \leq \beta^{-1} .
$$

Then for all initial values $x(0) \in \mathcal{X}_{\beta}$, the trajectories of $x(t)$ remain within $\mathcal{X}_{\beta}$, and the polytopic system representation (35) is valid. Hence $x(t)$ is a trajectory of the linear system (35) and $\dot{V}<0$ along the trajectories of the latter system which implies that $\lim _{t \rightarrow \infty} x(t)=0$.

Example 3. We consider (2) with the following matrices (taken from [3], where $h=0)$ :

$$
A=\left[\begin{array}{cc}
1.1 & -0.6 \\
.5 & -1
\end{array}\right], \quad B_{1}=\left[\begin{array}{l}
1 \\
1
\end{array}\right]
$$

and where $\bar{u}=5$. Applying Theorem 3.3 a stabilizing gain was obtained for all samplings with the maximum sampling interval $h \leq 0.75$. In order to 'enlarge' the volume of the ellipse we minimized the value of $\beta$ (to improve the result we also added the inequality $Q_{1}>\alpha I$ and chose such $\alpha>0$ that enlarged the resulting ellipse). The ellipse volume increases when $h$ decreases (see Figure 2). For, say, $h=0.75$ we obtain $K=[-1.69640 .5231]$ (with $\epsilon=0.325, \beta=0.1261, P_{1}=$ $\left[\begin{array}{cc}0.9132 & -0.2816 \\ -0.2816 & 0.0868\end{array}\right], \alpha=1$ ) and we show (see Figure 3 ) that a trajectory starting on

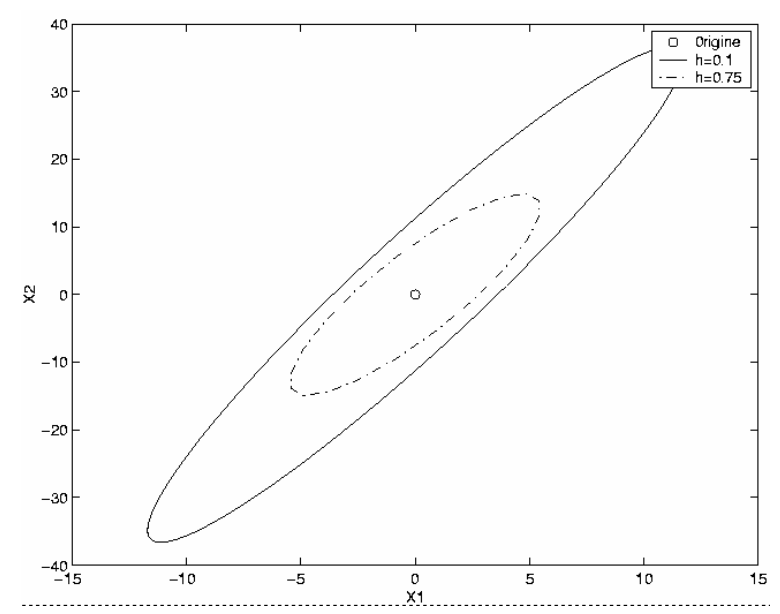

Fig. 2. Ellipsoidal bounds on the domain of attraction: line corresponds to $h=0.1$; slash line to $h=0.75$; point line to $h=$ 


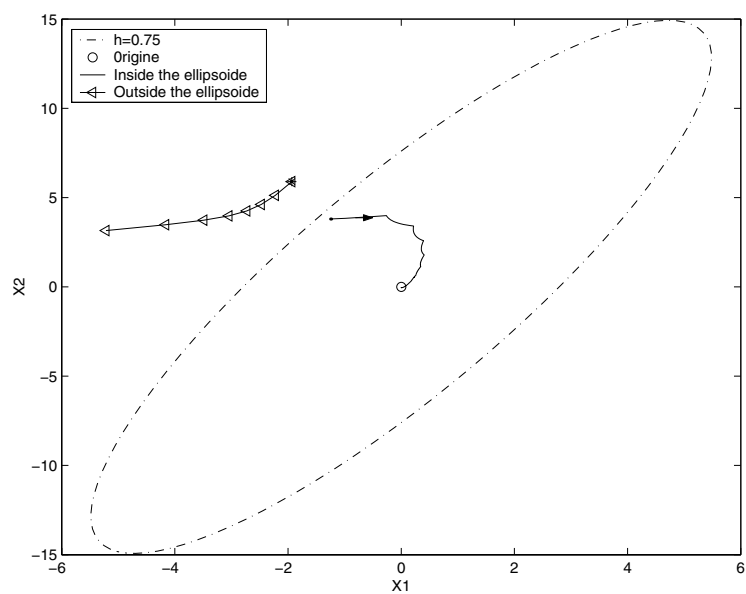

Fig. 3. Stabilization result for $h=0.75$

the periphery of the ellipse (for the case of the uniform sampling with the sampling period $\left.t_{k+1}-t_{k}=0.75\right)$ never leaves this ellipse and converges to the origin, while a trajectory starting not far from the ellipse remains outside the ellipse.

\section{Conclusions}

A new method for robust sampled-data stabilization of linear continuous-time systems is introduced. This method is based on the continuous-time model with time-varying input delay. Under assumption that the maximum sampling interval is not greater then $h>0$, the $h$-dependent sufficient LMIs conditions for stabilization of systems with polytopic type uncertainty and for regional stabilization of systems with sampled-data saturated state-feedback are derived via descriptor system approach to time-delay systems.

The new approach solves the problems for comparatively small $h$ and leads to sufficient conditions only, however these conditions are simple. The method may be applied to a wide spectrum of robust sampled-data control problems (e.g. to guaranteed cost or to $H_{\infty}$ control).

\section{References}

1. Astrom K., Wittenmark B. (1989) Adaptative Control. Prentice Hall publisher, Upper Saddle River

2. Bamieh B., Pearson J., Francis B., Tannenbaum A. (1991) A lifting technique for linear periodic systems. System \& Control Letters 17:79-88

3. Cao Y., Lin Z., Hu T. (2002) Stability analysis of linear time-delay systems subject to input saturation. IEEE Trans. on Circuits and Systems 49(2):233-240

4. Chen T., Francis B. (1991) $h_{2}$ optimal sampled-data control. IEEE Transactions on Automatic Control 36:387-397 
5. Dambrine M., Richard J.-P., Borne P. (1995) Feedback control of time-delay systems with bounded control and state. Mathematical Problems in Engineering, 1:77-87

6. Dullerud G., Glover K. (1993) Robust stabilization of sampled-data systems to structured lti perturbations. IEEE Transactions on Automatic Control 38:1497-1508

7. Fridman E. (1992) Use of models with aftereffect in the problem of design of optimal digital control. Automation and Remote Control 53:1523-1528

8. Fridman E. New lyapunov-krasovskii functionals for stability of linear retarded and neutral type systems. System \& Control Letters 43:309-319

9. Fridman E. Stability of linear descriptor systems with delay: A lyapunov-based approach. Journal of Mathematical Analysis and Applications 273(1):24-44

10. Fridman E., Pila A., Shaked U. (2003) Regional stabilization and control of timedelay systems with saturating actuators. International J. Robust Nonlinear Control 13(9):885-907

11. Fridman E., Shaked U. (2002) An improved stabilization method for linear timedelay systems. IEEE Trans. on Automatic Control 47(11):1931-1937

12. Fridman E., Shaked U. (2003) Parameter dependent stability and stabilization of uncertain time-delay systems. IEEE Transactions on Automatic Control 48(5):861-866

13. Gouaisbaut F., Dambrine M., Richard J.-P. (2002) Robust control of dealy systems: a sliding mode control design via lmi. Systems \& Control Letters 46(4):219-230

14. Hale J., Lunel S. (1993) Introduction of functional differential equations. SpringerVerlag, Berlin Heidelberg New York

15. Kim J.H. (2001) Delay and its time-derivative dependent robust stability of time-delayed linear systems with uncertainty. IEEE Trans. on Automatic Control 46:789-792

16. Kolmanovskii V., Myshkis A. (1999) Applied theory of functional differential equations. Kluwer, Dordrecht

17. Kolmanovskii V., Niculescu S.I., Richard J.-P. (1999) On the lyapunov-krasovskii functionals for stability analysis of linear delay systems. Int. J. Control 72:374-384

18. Li X., de Souza C. (1997) Criteria for robust stability and stabilization of uncertain linear systems with state delay. Automatica 33:1657-1662

19. Mikheev Yu.V., Sobolev V.A., Fridman E.M. (1988) Asymptotic analysis of digital control systems. Automation and Remote Control 49(9):1175-1180

20. Niculescu S.I. (2001) Delay effects on stability: A robust control approach. In Lecture Notes in Control and Information Sciences, Springer-Verlag, Berlin Heidelberg New York

21. Oishi Y. (1997) A bound of conservativeness in sampled-data robust stabilization and its dependence on sampling periods. Systems \& Control Letters 32:11-19

22. Osborn S., Bernstein D. (1995) An exact treatement of the achievable closed-loop $h_{2}$-performance of sampled-data controllers: From continuous-time to open-loop. Automatica 31(4):617-620

23. Takaba K., Morihira N., Katayama T. (1995) A generalized lyapunov theorem for descriptor systems. Systems \& Control Letters 24:49-51

24. Tarbouriech S., Gomes da Silva J. (2000) Synthesis of controllers for continuoustime delay systems with saturating controls via lmi's. IEEE transactions on $\mathrm{Au}-$ tomatic Control 45(1):105-111

25. Trentelman H., Stoorvogel A. (1995) Sampled-data and discrete-time $h^{2}$ optimal control. J. Control and Optimization 33(3):834-862

26. Yamomoto Y. (1990) New approach to sampled-data control systems - a function space method. In Proc. 29th Conf. on Decision and Control, pp. 1882-1887, Honolulu, Haiwai 